\title{
Silent cerebral infarcts in patients with sickle cell disease: a systematic review and meta-analysis
}

Maite E. Houwing ${ }^{1 *}$ (D, Rowena L. Grohssteiner ${ }^{1}$, Marjolein H. G. Dremmen², Ferdows Atiq ${ }^{3}$, Wichor M. Bramer ${ }^{4}$, Anne P. J. de Pagter ${ }^{1}$, C. Michel Zwaan ${ }^{1,5}$, Tonya J. H. White ${ }^{6,7}$, Meike W. Vernooij ${ }^{7,8}$ and Marjon H. Cnossen ${ }^{1}$

\begin{abstract}
Background and purpose: Silent cerebral infarcts $(\mathrm{SCls})$ are the most common neurological complication in children and adults with sickle cell disease (SCD). In this systematic review, we provide an overview of studies that have detected SCIs in patients with SCD by cerebral magnetic resonance imaging (MRI). We focus on the frequency of SCls, the risk factors involved in their development and their clinical consequences.

Methods: The databases of Embase, MEDLINE ALL via Ovid, Web of Science Core Collection, Cochrane Central Register of Trials via Wiley and Google Scholar were searched from inception to June 1, 2019.

Results: The search yielded 651 results of which 69 studies met the eligibility criteria. The prevalence of SCls in patients with SCD ranges from 5.6 to $80.6 \%$ with most studies reported in the 20 to $50 \%$ range. The pooled prevalence of SCls in $\mathrm{HbSS}$ and $\mathrm{HbS} \beta^{\circ}$ SCD patients is $29.5 \%$. SCls occur more often in patients with the HbSS and $\mathrm{HbS} \beta^{0}$ genotype in comparison with other SCD genotypes, as SCls are found in $9.2 \%$ of $\mathrm{HbSC}$ and $\mathrm{HbS}^{+}$patients. Control subjects showed a mean pooled prevalence of SCls of 9.8\%. Data from included studies showed a statistically significant association between increasing mean age of the study population and mean $\mathrm{SCl}$ prevalence. Thirty-three studies examined the risk factors for $\mathrm{SCls}$. The majority of the risk factors show no clear association with prevalence, since more or less equal numbers of studies give evidence for and against the causal association.
\end{abstract}

Conclusions: This systematic review and meta-analysis shows SCls are common in patients with SCD. No clear risk factors for their development were identified. Larger, prospective and controlled clinical, neuropsychological and neuroimaging studies are needed to understand how SCD and SCls affect cognition.

Keywords: Sickle cell disease, Silent cerebral infarction, Stroke, Magnetic resonance imaging

\footnotetext{
* Correspondence: m.houwing@erasmusmc.nl

'Department of Pediatric Haematology and Oncology, Erasmus MC - Sophia

Children's Hospital, NC-825, Wytemaweg 80, 3015 CN Rotterdam, The

Netherlands

Full list of author information is available at the end of the article
}

(c) The Author(s). 2020 Open Access This article is licensed under a Creative Commons Attribution 4.0 International License, which permits use, sharing, adaptation, distribution and reproduction in any medium or format, as long as you give appropriate credit to the original author(s) and the source, provide a link to the Creative Commons licence, and indicate if changes were made. The images or other third party material in this article are included in the article's Creative Commons licence, unless indicated otherwise in a credit line to the material. If material is not included in the article's Creative Commons licence and your intended use is not permitted by statutory regulation or exceeds the permitted use, you will need to obtain permission directly from the copyright holder. To view a copy of this licence, visit http://creativecommons.org/licenses/by/4.0/. The Creative Commons Public Domain Dedication waiver (http://creativecommons.org/publicdomain/zero/1.0/) applies to the data made available in this article, unless otherwise stated in a credit line to the data. 


\section{Background}

Sickle cell disease (SCD) is an autosomal recessive haemoglobinopathy characterized by ongoing haemolytic anaemia, episodes of vaso-occlusion and progressive organ failure. Millions are affected worldwide, and approximately 312.000 neonates with this haematological disorder are born annually [1]. SCD is caused by a single nucleotide substitution in codon 6 of the $\beta$-globin gene. This mutation leads to the formation of abnormal haemoglobin, called HbS [2]. When deoxygenated, HbS erythrocytes become sickle or crescent-shaped, rigid and prone to lysis. These sickle cells interact with leukocytes and the vascular endothelium causing occlusion and vasculopathy, subsequently leading to a broad range of acute and chronic complications including cerebrovascular disease [3, 4].

The most common neurological complication in children and adults with SCD is the development of silent cerebral infarcts (SCIs), also referred to as silent strokes [5-7]. In contrast to the clinically overt strokes, SCIs do not lead to apparent focal neurological symptoms and can only be detected with neuroimaging techniques [8, 9]. As a consequence, SCIs are identified incidentally or through screening. Although SCIs do not lead to any tangible motor or sensory deficits, they are associated with cognitive morbidity and an increased risk of future strokes [10-12]. SCIs are visible as focal lesions on both computed tomography (CT) scans and magnetic resonance imaging (MRI) scans. Detection is however better by MRI due to the greater range of contrast between soft tissues and greater detail in the depiction of intracranial structures [13].

There is an ongoing debate over the rationale of screening for SCIs in patients with SCD [14, 15]. While the Silent Cerebral Infarct Transfusion (SIT) randomized controlled trial showed that chronic red blood cell transfusions reduce the risk of recurrent infarction, this benefit was incomplete with some children in the transfusion therapy arm also developing infarct recurrence [16]. More importantly, the true incidence and prevalence of SCIs remain unknown and the understanding of the pathophysiology and risk factors limited. In this systematic review, we provide an overview of studies that have used brain MRI studies to detect SCIs in patients with SCD while focusing on the frequency of SCIs, the risk factors potentially involved in their occurrence and their clinical impact. Emphasis is placed on the epidemiology of SCIs and not on the evaluation of intervention studies.

\section{Methods}

\section{Article retrieval}

For this report, the Preferred Reporting Items for Systematic Reviews and Meta-Analyses (PRISMA) guidelines were followed [17]. A comprehensive systematic search was performed in Embase, MEDLINE ALL via Ovid, Web of Science Core Collection, Cochrane Central Register of Trials via Wiley and Google Scholar (Additional file 1) from inception to June 1, 2019. Search terms included multiple synonyms for 'SCIs', 'SCD' and 'MRI' in various combinations. No limitations in the search strategy were inserted. The search strategy was designed and conducted by an experienced librarian (W.M.B.) with input from the primary investigators.

\section{Study selection}

Studies were screened on potential eligibility by two independent reviewers (M.E.H., R.L.G.). Papers had to be written in English. Studies had to report original data; reviews, case reports and letters were excluded. Both controlled studies and (retrospective) cohort studies were eligible. Studies were included if they involved patients of all ages with either homozygous or compound heterozygous SCD without overt stroke and specifically assessed for detection of SCIs by MRI. Differences of opinion were resolved by discussion and consensus between the reviewers.

\section{Data assessment}

For each included study, the following information was collected: study design, characteristics of the patient population, mean or median age at study inclusion, utilized MRI protocol, additionally performed advanced MR and ultrasonic imaging techniques, SCI prevalence, risk factors studied, clinical consequences and other major observations or conclusions. For any missing information or unresolved discrepancies, we contacted the authors of the studies for clarification or to request unpublished data. As it is difficult to determine whether a focal hyperintensity seen on MRI is caused by actual infarction or by another underlying cause (e.g. inflammation, infection, demyelination) [18], all different terms used for strongly related MRI findings in included studies (e.g. white matter hyperintensities, white matter changes, silent lesions, ischaemic lesions) were considered to be SCIs. The reviewers read and abstracted each article, and a third member with specific imaging expertise (M.H.D) checked the table entries for accuracy with regard to the original articles. Data were reviewed descriptively.

Where multiple articles were included for a single or overlapping population sample, prevalence and incidence estimates were obtained from the report with the largest sample size to prevent duplication.

\section{Statistical methods}

Continuous data are presented as mean and range or as 95\% confidence intervals (CI), whereas categorical data 
are presented as frequency and proportion (\%). The mean pooled prevalence of SCIs was calculated with univariate general linear models, in which we weighted SCI prevalence for the sample size of the included studies. The outcomes are presented as pooled mean prevalence and $95 \% \mathrm{CI}$. We also used univariate general linear models to compare the mean pooled prevalence of SCIs between the two independent groups.

The association between the prevalence of SCIs and age was analysed with linear regression analysis. We adjusted the outcomes of the linear regression analysis for publication year, study design (i.e. prospective cohort study or retrospective cohort study), sample size and field strength. The outcomes of the linear regression analysis are presented as unstandardized beta $(\beta), 95 \%$ $\mathrm{CI}$ and $p$ value.

For studies that did not report the mean age of study participants, grouped data mean calculation formula was used to calculate the mean. For open-end age intervals (e.g. $<25$ or $>50$ years) where it is not possible to calculate the mean, the median and calculated median of study participants were applied.

\section{Risk factor analysis}

Selected articles were evaluated to identify all studied risk factors. The reported statistics described in univariate analyses were examined to determine the direction of the association of a particular risk factor and whether it was deemed statistically significant. 'Independent' risk factors were identified from studies in which multivariable analyses were conducted.

\section{Results}

The literature search yielded a total of 651 non-duplicate citations that were screened using predetermined inclusion and exclusion criteria. A total of 69 full-text articles met the inclusion criteria for this review. These publications reported data on the frequency of SCIs in patients with SCD from 41 studies. Figure 1 shows the flow chart of articles resulting from the initial search to the final inclusion or exclusion. Twenty-three studies were conducted in the USA (58.5\%), 14 in Europe, one in Brazil, one in India, one in Turkey and one in Kuwait.

\section{Definitions of SCls and MRI detection}

An important finding was the general lack of uniformity in definitions of SCIs in SCD. Definitions identified were based on both MRI criteria and clinical characteristics (e.g. normal neurological examination). In most studies, SCIs were defined as focal areas of abnormally increased T2-weighted signal intensity on multiple anatomical views without associated neurological deficits. Approximately half of the studies applied a more precise definition in which a focal brain lesion was required to be at least 3 or $5 \mathrm{~mm}$ in one dimension and visible in two planes on fluid-attenuated inversion recovery (FLAI R) T2-weighted images, with a normal neurological examination. Eight studies did not explicitly state what the applied definition for SCIs was.

In addition to the varying definitions of SCIs, the detection of SCIs depends on MRI parameters including magnet strength and spatial resolution (most importantly slice thickness). The majority of studies (72\%) used a 1.5-T MRI magnet, a 3.0-T magnet was used in $17 \%$ of studies and the remaining $11 \%$ used another magnet strength (i.e. $0.6 \mathrm{~T}, 1.0 \mathrm{~T}, 7.0 \mathrm{~T}$ ). Slice thickness varied from 1.0 to $6.0 \mathrm{~mm}$ and was not mentioned in 24 (58.5\%) studies.

Information concerning MRI parameters-including magnet strength and sequences-as well as imaging and clinical criteria used for diagnosis of SCIs, in the 41 included studies, is depicted in Table 1.

\section{Additional neuroimaging techniques Transcranial Doppler}

The majority of studies excluded patients with abnormal transcranial Doppler (TCD) flow velocities $(>170 \mathrm{~cm} / \mathrm{s})$ indicative of an increased risk of overt stroke. Most studies that concomitantly measured TCD velocities found no significant differences between the mean TCD velocities of patients with normal MRI scans and patients with detected SCIs [6, 23, 57-64]. Moreover, comparison of patients who were included in both the Cooperative Study of SCD (CSSCD) and the Stroke Prevention (STOP) trial showed that patients with abnormal TCDs did not demonstrate a concurrent high prevalence of SCIs. Conversely, those who had SCIs did not present with a high prevalence of abnormally increased TCD velocities [64].

In contrast, two studies-one retrospective cohort study and one prospective cohort study-in respectively 254 and 23 SCD patients reported a significant association between higher (maximum) TCD velocities and SCIs $[24,55]$.

\section{Magnetic resonance angiography}

More than half of the included studies performed magnetic resonance angiography (MRA) as part of the MRI examination. Some studies found that signs of MRAdefined cerebral vasculopathy were related to the presence and/or number of SCIs $[6,20,24,58,63]$. In the Silent Cerebral Infarct Transfusion (SIT) trial, the frequency of intracranial vasculopathy in patients with and without SCIs was $15.9 \%$ and $6.3 \%$, respectively ( $p<$ $0.001)$. However, the majority $(84 \%)$ of patients with SCIs did not show vasculopathy on MRA [63]. 


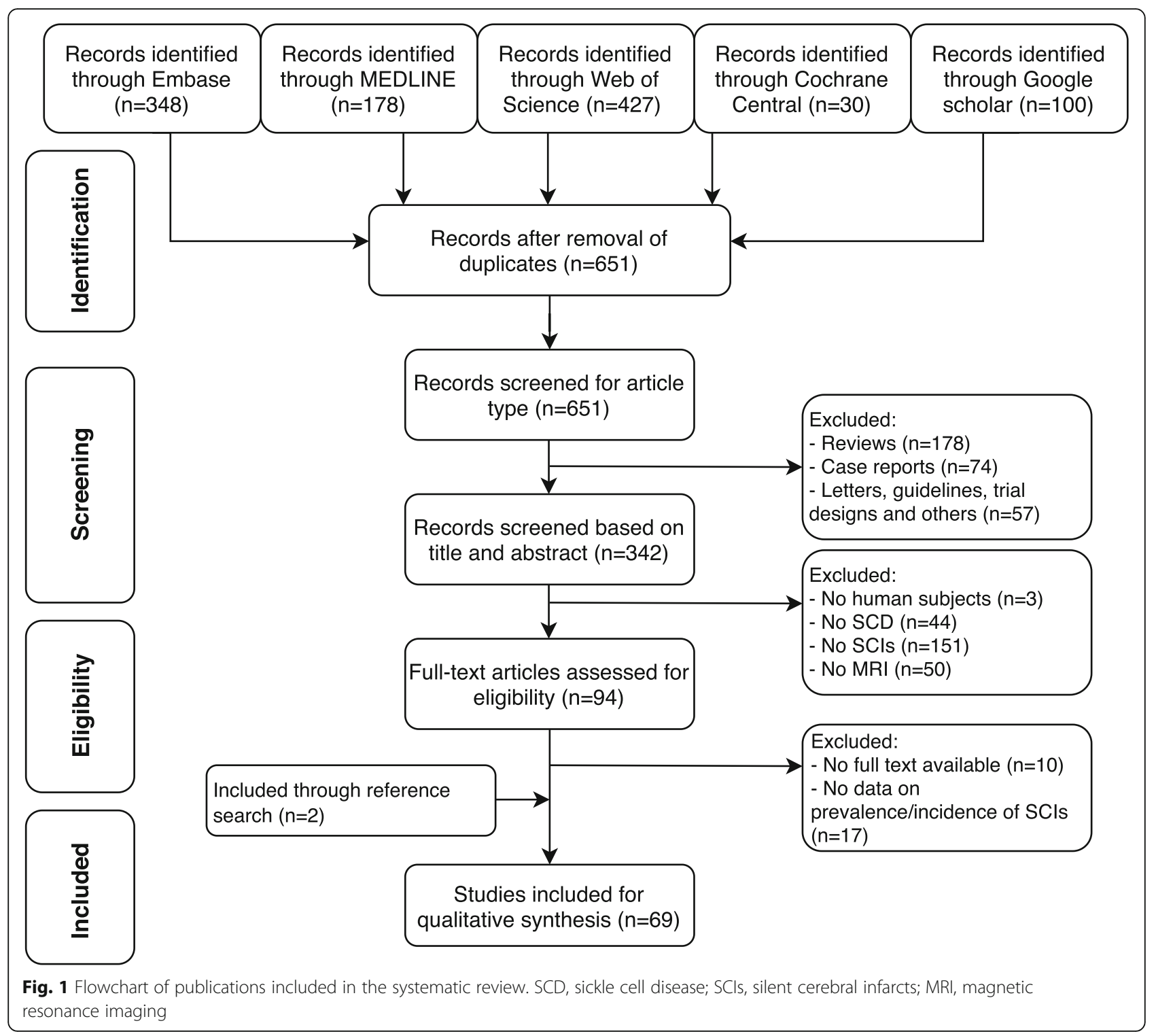

\section{Arterial spin labelling}

Arterial spin labelling (ASL) provides a method to noninvasively obtain a quantitative measurement of cerebral blood flow. The majority of recently performed ASL studies confirm an elevated global cerebral blood flow in patients with $\mathrm{SCD}$, with no differences between patients with and without SCIs [40, 52, 65, 66]. However, a study by Ford et al. compared cerebral blood flow maps from children with and without SCIs and found that SCIs were associated with impaired haemodynamics including low cerebral blood flow in the region of the highest infarct density $(p<0.001)$ [31].

\section{$\mathrm{SCl}$ localization}

Approximately $80 \%$ of children in whom SCIs were detected in the CSSCD study had abnormalities in the frontoparietal deep white matter and periventricular regions on MRI, with other infarcts located in the basal ganglia and the temporal lobe. Infarcts were distributed equally in both brain hemispheres $[8,10]$. Similar observations were reported in other studies $[20,22,28,30,33$, $50,55,59,62]$. Both overt strokes and SCIs predominantly occurred in the watershed regions of the deep white matter and encompassed only $5.6 \%$ of the brain volume [31].

\section{$\mathrm{SCl}$ frequency}

The prevalence and incidence of SCIs in patients with SCD varied widely, depending on the study population and MRI protocol. While most included studies were cohort studies, both prospective and retrospective, some case-control studies and one randomized controlled trial 
Table 1 Applied definitions and magnetic resonance imaging parameters in studies of silent cerebral infarcts in sickle cell disease patients

\begin{tabular}{|c|c|c|c|c|c|}
\hline Author (year) & $\begin{array}{l}\text { Magnet } \\
\text { strength }\end{array}$ & $\begin{array}{l}\text { Slice } \\
\text { thickness } \\
(\mathrm{mm})\end{array}$ & Sequence & MRI criteria for SCls detection & Clinical criteria for SCls \\
\hline $\begin{array}{l}\text { Abboud et al. } \\
2011[19]\end{array}$ & - & 5.0 & $\begin{array}{l}\text { T1: ax | FLAIR: ax, } \\
\text { cor }\end{array}$ & Evidence of cerebral infarction on MRI & No history of overt stroke \\
\hline $\begin{array}{l}\text { Arkuszewski } \\
\text { et al. } 2014 \text { [20] }\end{array}$ & $3.0 \top$ & - & FLAIR & $\begin{array}{l}\text { Area of abnormal hyperintensity, } \\
\geq 3 \mathrm{~mm} \text { on FLAIR, visible on at least } \\
\text { two perpendicular planes }\end{array}$ & - \\
\hline $\begin{array}{l}\text { Asbeutah et al. } \\
2014[21]\end{array}$ & $1.5 \mathrm{~T}$ & 5.0 & $\begin{array}{l}\text { T1: ax, sag | T2: } \\
\text { ax, sag | FLAIR }\end{array}$ & - & - \\
\hline $\begin{array}{l}\text { Baldeweg et al. } \\
2006 \text { [22] }\end{array}$ & $1.5 \mathrm{~T}$ & - & $\begin{array}{l}\text { T1: sag, cor | T2: } \\
\text { ax | FLAIR: cor }\end{array}$ & $\begin{array}{l}\text { Area of abnormal hyperintensity on } \\
\text { T2 }\end{array}$ & $\begin{array}{l}\text { No history of a focal neurological deficit } \\
\text { lasting }>24 \mathrm{~h}\end{array}$ \\
\hline $\begin{array}{l}\text { Bernaudin et al. } \\
2011 \text { [23] }\end{array}$ & 1.0 or $1.5 \mathrm{~T}$ & - & $\mathrm{T} 1|\mathrm{~T} 2| \mathrm{FLAIR}$ & $\begin{array}{l}\text { Signal abnormality, } \geq 3 \mathrm{~mm} \text { in one } \\
\text { dimension, visible on two views on } \mathrm{T} 2\end{array}$ & $\begin{array}{l}\text { No history of overt stroke and normal } \\
\text { neurologic examination }\end{array}$ \\
\hline $\begin{array}{l}\text { De Blank et al. } \\
2010[24]\end{array}$ & - & - & - & Evidence of cerebral infarction on MRI & No overt neurological symptoms \\
\hline $\begin{array}{l}\text { Brousse et al. } \\
2017 \text { [25] }\end{array}$ & - & - & - & - & - \\
\hline $\begin{array}{l}\text { Brown et al. } \\
2000 \text { [26] }\end{array}$ & - & - & - & Evidence of cerebral infarction on MRI & $\begin{array}{l}\text { No history of overt stroke and normal } \\
\text { neurological examination }\end{array}$ \\
\hline $\begin{array}{l}\text { Calvet et al. } \\
2017[27]\end{array}$ & $3.0 \mathrm{~T}$ & - & $\mathrm{T} 2 \mid \mathrm{FLAIR}$ & $\begin{array}{l}\text { White matter lesions, i.e. poorly } \\
\text { defined hyperintensities, } \geq 5 \mathrm{~mm} \text { on } \\
\text { T2 or FLAIR }\end{array}$ & $\begin{array}{l}\text { No history of overt stroke or overt } \\
\text { neurological symptoms }\end{array}$ \\
\hline $\begin{array}{l}\text { Coloigner et al. } \\
2017 \text { [28] }\end{array}$ & $3.0 \top$ & $\begin{array}{l}1.0 \text { or } 1.3 \\
\text { or } 5.0\end{array}$ & 3D T1 | 3D T2 | & $\begin{array}{l}\geq 3 \mathrm{~mm} \text { lesions on } 3 \mathrm{D} \text { T2 observed in } \\
\text { two orthogonal planes }\end{array}$ & No history of overt stroke \\
\hline $\begin{array}{l}\text { Dowling et al. } \\
2012 \text { [29] }\end{array}$ & 1.5 or $3.0 \top$ & - & FLAIR: ax & $\begin{array}{l}\text { Area of abnormal hyperintensity } \\
\text { intensity in multiple T2 }\end{array}$ & $\begin{array}{l}\text { No history or physical findings of a } \\
\text { focal neurological deficit lasting }>24 \mathrm{~h}\end{array}$ \\
\hline $\begin{array}{l}\text { Ford et al. } \\
2017 \text { [30] }\end{array}$ & - & - & $\mathrm{T} 1$ |FLAIR & $\begin{array}{l}\text { Area of abnormal hyperintensity on } \\
\text { FLAIR }>3 \mathrm{~mm} \text { and cerebrospinal fluid- } \\
\text { like hypointensity on } \mathrm{T1}\end{array}$ & - \\
\hline $\begin{array}{l}\text { Ford et al. } \\
2018 \text { [31] }\end{array}$ & - & - & T1 |FLAIR & $\begin{array}{l}\text { Signal abnormality, } \geq 3 \mathrm{~mm} \text { in one } \\
\text { dimension, visible on two planes on } \\
\text { FLAIR T2 }\end{array}$ & $\begin{array}{l}\text { Normal neurological examination or } \\
\text { absence of neurological symptoms that } \\
\text { correlate with lesion location }\end{array}$ \\
\hline $\begin{array}{l}\text { Gold et al. } \\
2008 \text { [32] }\end{array}$ & - & - & - & - & - \\
\hline $\begin{array}{l}\text { Guilliams et al. } \\
2015 \text { [33] }\end{array}$ & 1.5 or $3.0 \mathrm{~T}$ & 5.0 & $\begin{array}{l}\text { T1: sag | T2: ax } \\
\text { |FLAIR: ax, cor }\end{array}$ & $\begin{array}{l}\text { Signal abnormality, } \geq 3 \mathrm{~mm} \text { in one } \\
\text { dimension, visible on two planes on } \\
\text { T2 or FLAIR }\end{array}$ & $\begin{array}{l}\text { Absence of neurological symptoms that } \\
\text { correlate with lesion location }\end{array}$ \\
\hline $\begin{array}{l}\text { Gyang et al. } \\
2011 \text { [34] }\end{array}$ & - & - & - & Abnormal MRI changes & No neurological symptoms \\
\hline $\begin{array}{l}\text { Issar et al. } \\
2018 \text { [35] }\end{array}$ & $1.5 \mathrm{~T}$ & - & $\begin{array}{l}\text { T1: ax | T1 FLAIR: } \\
\text { sag | T2: ax } \\
\text { IFLAIR: ax }\end{array}$ & $\begin{array}{l}\text { Areas of abnormal hyperintensity on } \\
\text { FLAIR T2 }\end{array}$ & $\begin{array}{l}\text { Absence of overt clinical neurological } \\
\text { symptoms }\end{array}$ \\
\hline $\begin{array}{l}\text { Kassim et al. } \\
2016[36]\end{array}$ & - & - & $\mathrm{T} 2$ & $\geq 3 \mathrm{~mm}$ on $\mathrm{T} 2$ in two imaging planes & $\begin{array}{l}\text { No history of neurological deficits and } \\
\text { normal neurological examination }\end{array}$ \\
\hline $\begin{array}{l}\text { Kawadler et al. } \\
2018 \text { [37] }\end{array}$ & $1.5 \mathrm{~T}$ & - & T2: ax & - & - \\
\hline $\begin{array}{l}\text { Kwiatkowski } \\
\text { et al. } 2009 \text { [6] }\end{array}$ & $1.5 \mathrm{~T}$ & - & FLAIR & $\begin{array}{l}\text { Area of abnormal hyperintensity on } \\
\text { T2 and FLAIR }\end{array}$ & $\begin{array}{l}\text { No history of overt stroke or motor } \\
\text { deficits that can be attributed to the } \\
\text { lesion }\end{array}$ \\
\hline $\begin{array}{l}\text { Melek et al. } \\
2006 \text { [38] }\end{array}$ & $1.5 \mathrm{~T}$ & 5.0 & $\begin{array}{l}\text { T1: sag, ax | T2: } \\
\text { sag, ax |PD: ax }\end{array}$ & Abnormal MRI & $\begin{array}{l}\text { No history or physical findings of a } \\
\text { focal neurological deficit lasting }>24 \mathrm{~h}\end{array}$ \\
\hline $\begin{array}{l}\text { Mercuri et al. } \\
1995 \text { [39] }\end{array}$ & $1.0 \mathrm{~T}$ & 6.0 & $\mathrm{~T} 2$ & - & - \\
\hline $\begin{array}{l}\text { Oguz et al. } \\
2003 \text { [40] }\end{array}$ & $1.5 \mathrm{~T}$ & 5.0 & $\begin{array}{l}\text { T1: sag | T2: ax } \\
\text { |FLAIR }\end{array}$ & - & - \\
\hline
\end{tabular}


Table 1 Applied definitions and magnetic resonance imaging parameters in studies of silent cerebral infarcts in sickle cell disease patients (Continued)

\begin{tabular}{|c|c|c|c|c|c|}
\hline Author (year) & $\begin{array}{l}\text { Magnet } \\
\text { strength }\end{array}$ & $\begin{array}{l}\text { Slice } \\
\text { thickness } \\
(\mathrm{mm})\end{array}$ & Sequence & MRI criteria for SCls detection & Clinical criteria for SCls \\
\hline $\begin{array}{l}\text { Onofri et al. } \\
2012[41]\end{array}$ & $1.5 \mathrm{~T}$ & 5.0 & FLAIR: ax & - & - \\
\hline $\begin{array}{l}\text { Pegelow et al. } \\
2001 \text { [42] }\end{array}$ & - & 5.0 & $\begin{array}{l}\mathrm{T} 1: \mathrm{ax} \mid \mathrm{PD} \\
\mathrm{T} 2: \mathrm{ax}, \mathrm{cor}\end{array}$ & - & - \\
\hline $\begin{array}{l}\text { Pegelow et al. } \\
2002[10]\end{array}$ & - & - & - & Abnormal MRI & No neurological deficit \\
\hline $\begin{array}{l}\text { Quinn et al. } \\
2013 \text { [43] }\end{array}$ & - & - & FLAIR & $\begin{array}{l}\text { Signal abnormality } \geq 3 \mathrm{~mm} \text { in one } \\
\text { dimension, visible on at least two } \\
\text { views of } \mathrm{T} 2 \text { FLAIR }\end{array}$ & $\begin{array}{l}\text { Normal neurological examination or } \\
\text { absence of neurological symptoms that } \\
\text { correlate with lesion location }\end{array}$ \\
\hline $\begin{array}{l}\text { Schatz et al. } \\
2006[44]\end{array}$ & $1.5 \mathrm{~T}$ & 5.0 & $\begin{array}{l}\text { T1: sag | T2: } \\
\text { ax }\end{array}$ & $\begin{array}{l}\text { Area of abnormal hyperintensity, } \\
\geq 3 \mathrm{~mm} \text { on } \mathrm{T} 2\end{array}$ & Normal neurological history \\
\hline $\begin{array}{l}\text { Seibert et al. } \\
1993 \text { [45] }\end{array}$ & $1.5 \mathrm{~T}$ & - & $\begin{array}{l}\mathrm{T} 1|\mathrm{~T} 2| \mathrm{PD}: \\
\operatorname{ax}\end{array}$ & - & - \\
\hline $\begin{array}{l}\text { Silva et al. } \\
2009 \text { [46] }\end{array}$ & $1.5 \mathrm{~T}$ & 5.0 & $\begin{array}{l}\text { T1: sag, ax }|\mathrm{T} 2| \\
\text { FLAIR: ax }\end{array}$ & $\begin{array}{l}\text { Evidence of ischaemia, including } \\
\text { lacunar infarction, encephalomalacia, } \\
\text { atrophy or leukoencephalopathy }\end{array}$ & - \\
\hline $\begin{array}{l}\text { Solomou et al. } \\
2013[47]\end{array}$ & $1.0 \mathrm{~T}$ & 5.0 & $\begin{array}{l}\text { T2: ax, cor | } \\
\text { FLAIR: ax, cor }\end{array}$ & $\begin{array}{l}\text { Focal }(<1 \mathrm{~cm}) \text { or multiple }(>1 \mathrm{~cm}) \\
\text { high-intensity lesions on T2 or FLAIR }\end{array}$ & $\begin{array}{l}\text { Absence of physical findings of overt } \\
\text { stroke }\end{array}$ \\
\hline $\begin{array}{l}\text { Steen et al. } \\
2003 \text { [48] }\end{array}$ & $1.5 \mathrm{~T}$ & 5.0 or 3.0 & $\begin{array}{l}\text { T1: ax | T2: } \\
\text { ax |FLAIR: ax }\end{array}$ & $\begin{array}{l}\text { Evidence of ischaemia, including } \\
\text { lacunar infarction, encephalomalacia, } \\
\text { atrophy or leukoencephalopathy }\end{array}$ & Absence of a clinical history of stroke \\
\hline $\begin{array}{l}\text { Tewari et al. } \\
2018 \text { [49] }\end{array}$ & - & - & FLAIR & $\begin{array}{l}\text { Area of abnormal hyperintensity } \\
\geq 3 \mathrm{~mm} \text { in diameter and visible in at } \\
\text { least two planes of } \mathrm{T} 2 \text { (ax and cor) }\end{array}$ & $\begin{array}{l}\text { No history or physical findings of a } \\
\text { focal neurological deficit in a } \\
\text { corresponding localizing vascular } \\
\text { distribution }\end{array}$ \\
\hline $\begin{array}{l}\text { Václavů et al. } \\
2019 \text { [50] }\end{array}$ & $3.0 \mathrm{~T}$ & - & 3D FLAIR & Multiple (> 1) hyperintensities $\geq 5 \mathrm{~mm}$ & - \\
\hline $\begin{array}{l}\text { Van der Land } \\
\text { et al. } 2015 \text { [51] }\end{array}$ & 3.0 and $7.0 \mathrm{~T}$ & - & T1 |FLAIR: ax & Areas of abnormal hyperintensity & $\begin{array}{l}\text { No history or physical findings of a } \\
\text { focal neurological deficit }\end{array}$ \\
\hline $\begin{array}{l}\text { Van der Land } \\
\text { et al. } 2016 \text { [52] }\end{array}$ & $3.0 \mathrm{~T}$ & 5.0 & T2 |FLAIR & $\begin{array}{l}\text { Hyperintensity of variable size in the } \\
\text { white matter on FLAIR, without } \\
\text { cavitation }\end{array}$ & $\begin{array}{l}\text { No history or physical findings of a } \\
\text { focal neurological deficit }\end{array}$ \\
\hline $\begin{array}{l}\text { Vichinsky et al. } \\
2010 \text { [53] }\end{array}$ & $1.5 \mathrm{~T}$ & - & $\mathrm{T} 1|\mathrm{~T} 2| \mathrm{PD}$ & $\begin{array}{l}\text { Area of abnormal hyperintensity at } \\
\text { least } 5 \mathrm{~mm} \text { on } \mathrm{T} 2 \text { and PD, with } \\
\text { corresponding hypointensity on } \mathrm{T} 1\end{array}$ & - \\
\hline $\begin{array}{l}\text { Wang et al. } \\
1998[54]\end{array}$ & $1.5 \mathrm{~T}$ & 5.0 & T1: $\mathrm{ax} \mid \mathrm{T} 2: \mathrm{ax}$ & $\begin{array}{l}\text { Area of abnormal hyperintensity } \\
\text { on T2 }\end{array}$ & $\begin{array}{l}\text { No history of neurological symptoms } \\
\text { compatible with overt stroke }\end{array}$ \\
\hline $\begin{array}{l}\text { Wang et al. } \\
2008 \text { [55] }\end{array}$ & $1.5 \mathrm{~T}$ & 5.0 & $\begin{array}{l}\text { T1: ax |FLAIR: } \\
\text { ax, cor }\end{array}$ & $\begin{array}{l}\text { Area of abnormal hyperintensity on } \\
\mathrm{T} 2 \text {, consistent with an ischaemic } \\
\text { lesion in white matter }\end{array}$ & - \\
\hline $\begin{array}{l}\text { Watkins et al. } \\
1998 \text { [56] }\end{array}$ & $1.5 \mathrm{~T}$ & 5.0 & T2: $a x$ & - & - \\
\hline $\begin{array}{l}\text { Zafeiriou et al. } \\
2004 \text { [57] }\end{array}$ & $1.5 \mathrm{~T}$ & & T1: $a x$ & $\begin{array}{l}\text { Area of abnormal hyperintensity on } \\
\text { T2 }\end{array}$ & - \\
\hline
\end{tabular}

MRI magnetic resonance imaging, SCls silent cerebral infarctions, ax axial, cor coronal, sag sagittal, $T 1$ T1-weighted, T2 T2-weighted, FLAIR fluid-attenuated inversion recovery, $P D$ proton density

were also included. We examined both prevalence (i.e. proportion of patients with SCIs at one particular time) and incidence (i.e. rate at which patients develop SCIs over time). One study in 23 individuals found a SCI prevalence rate that extremely deviated from the prevalences observed in other included studies, with a $Z$-score of 2.7 [51]. The prevalence of SCIs was most probably much higher in this study, due to the use of a 7-T MRI scan. To not distort the results, this study was deemed an outlier and excluded from frequency analyses. 


\section{Prevalence of $\mathrm{SCl}$ in $\mathrm{HbSS}$ and $\mathrm{HbS} \beta^{\circ}$ genotypes}

Most studies $(n=27)$ were performed in patients with $\mathrm{HbSS}$ and $\mathrm{HbS} \beta^{0} \mathrm{SCD}(n=2789)$, with overall prevalence rates ranging from 5.6 to $80.6 \%$ (Table 2). The pooled prevalence of SCIs in $\mathrm{HbSS}$ and $\mathrm{HbS} \beta^{0}$ genotypes was $29.5 \%$ (95\% CI 25.2-33.9).

Data from the included studies showed a statistically significant association between increasing mean age of the study population and mean SCI prevalence: $\beta=1.0 \%$ increase in SCI prevalence for 1 year increase in mean age (95\% CI 0.5-1.6), $p=0.001$, Fig. 2. When corrected for publication year, study design (i.e. prospective cohort study or retrospective cohort study), sample size and field strength, the association between age and SCI prevalence remained significant: $\beta=0.8 \%$ (95\% CI $0.2-$ 1.5), $p=0.012$.
Prevalence of SCls in other genotypes and healthy controls Twelve studies separately reported the prevalence of SCIs in other SCD genotypes, i.e. $\mathrm{HbSC} \mathrm{HbS}^{+}$(total $n=254)$, and in healthy controls $(n=266)[10,21,22$, $26,28-30,33,35,40,50,53]$. The mean pooled prevalence of SCIs was 9.2\% (95\% CI 2.9-15.4) in patients with $\mathrm{HbSC}$ and $\mathrm{HbS}^{+}$, which was significantly lower compared to patients with $\mathrm{HbSS}$ and $\operatorname{HbS} \beta^{0} \operatorname{SCD}(p=$ 0.006, Fig. 3).

In nine studies (total $n=266$ ), the prevalence of SCIs was reported for controls [21, 22, 28-30, 35, 40, 50, 53]. Control subjects were mostly matched adolescents or young adult family members, with a mean age varying between 9.8 and 37.4 years. Although healthy, information regarding possible $\mathrm{HbS}$ carrier status was not provided for all controls. Sickle cell trait (HbAS genotype)

Table 2 Prevalence of silent cerebral infarcts in $\mathrm{HbSS}$ and $\mathrm{HbS} \beta^{0}$ sickle cell disease patients

\begin{tabular}{|c|c|c|c|c|c|}
\hline Author (year) & Study design & Sample size & Mean age (years) & Field strength $(\mathrm{T})$ & Prevalence SCls (\%) \\
\hline Abboud et al. 2011 [19] & PCS & 77 & 12.3 & NA & 26.6 \\
\hline Arkuszewski et al. 2014 [20] & PCS & 67 & 8.8 & 3.0 & 37.7 \\
\hline Asbeutah et al. 2014 [21] & PCS & 40 & $10.1^{*}$ & 1.5 & 10 \\
\hline de Blank et al. 2010 [24] & RCS & 254 & 10.6 & NA & 30.7 \\
\hline Brousse et al. 2017 [25] & PCS & 59 & 11.4 & NA & 13.6 \\
\hline Brown et al. 2000 [26] & PCS & 48 & $9.8^{*}$ & NA & 22.9 \\
\hline Calvet et al. 2017 [27] & RCS & 83 & 43.3 & 3.0 & 49.4 \\
\hline Ford et al. 2017 [30] & PCS & 22 & 27 & NA & 54.5 \\
\hline Ford et al. 2018 [31] & PCS & 1061 & NA & NA & 27 \\
\hline Guilliams et al. 2015 [33] & RCS & 168 & NA & $1.5 / 3.0$ & 27.4 \\
\hline Gyang et al. 2011 [34] & RCS & 8 & 15 & NA & 50 \\
\hline Kassim et al. 2016 [36] & RCS & 60 & 30 & NA & 53.3 \\
\hline Kwiatkowski et al. 2009 [6] & RCS & 65 & 3.6 & 1.5 & 27.7 \\
\hline Marouf et al. 2003 [67] & PCS & 35 & 26.9 & NA & 20 \\
\hline Mercuri et al. 1995 [39] & PCS & 11 & 9.3 & 1.0 & 45.5 \\
\hline Nottage et al. 2016 [68] & PCS & 50 & 9.4 & NA & 38 \\
\hline Oguz et al. 2003 [40] & PCS & 18 & 8.7 & 1.5 & 5.6 \\
\hline Pegelow et al. 2002 [10] & RCS & 266 & $8.3^{*}$ & NA & 21.8 \\
\hline Schatz et al. 2005 [44] & PCS & 20 & $12.2^{* *}$ & 1.5 & 40 \\
\hline Silva et al. 2009 [46] & PCS & 46 & 26.8 & 1.5 & 56.5 \\
\hline Tewari et al. 2018 [49] & PCS & 51 & $12.4^{* *}$ & NA & 37.3 \\
\hline Václavů et al. 2019 [50] & PCS & 36 & 31.9 & 3.0 & 80.6 \\
\hline Van der Land et al. 2015 [51] & PCS & 10 & 23 & $3.0 / 7.0$ & 50 \\
\hline Van der Land et al. 2016 [52] & PCS & 34 & 12.1 & 3.0 & 41.2 \\
\hline Vichinsky et al. 2010 [53] & PCS & 141 & 31.6 & 1.5 & 28.9 \\
\hline Wang et al. 1998 [54] & PCS & 36 & 1.5 & 1.5 & 8.3 \\
\hline Wang et al. 2008 [55] & PCS & 23 & 1.1 & 1.5 & 13 \\
\hline
\end{tabular}

SCls silent cerebral infarctions, PCS prospective cohort study (including case-control and randomized controlled trial), $R C S$ retrospective cohort study, NA not available

*Only the mean age for the full sample was provided, not separately for $\mathrm{HbSS}$ and $\mathrm{HbS} \beta^{0}$ thalassemia patients

**Only the mean age for the $\mathrm{SCl}$-affected and $\mathrm{SCl}$-unaffected group separately was provided, not for the full sample 


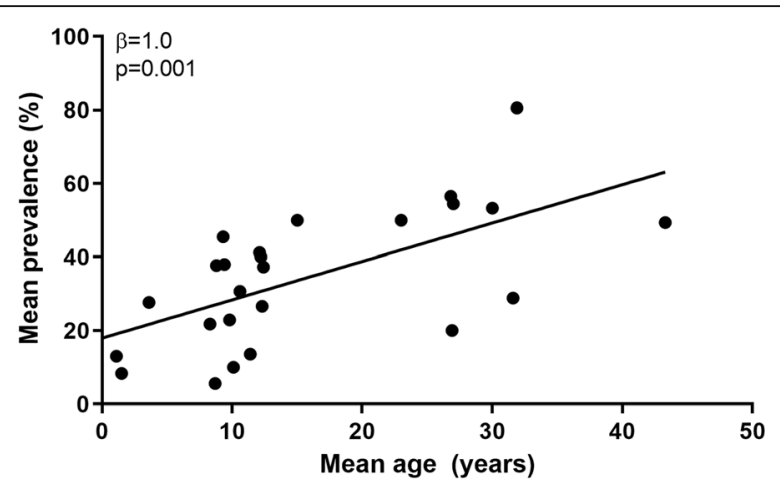

Fig. 2 The mean prevalence of silent cerebral infarcts, by mean age in patients with $\mathrm{HbSS}$ and $\mathrm{HbS}^{0}$ sickle cell disease. Linear regression analysis was used to analyse the association between age and $\mathrm{SCl}$ prevalence

was reported in 40 control subjects, whereas 132 subjects were explicitly reported not to have sickle cell trait (i.e. HbAA). Carrier status was unknown for the remaining 94 controls. Additional Table 1 shows the prevalence of SCIs and information regarding carrier status for control subjects. Control subjects showed a mean pooled prevalence of SCIs of 9.8\% (95\% CI - 0.520.1). Surprisingly, there was no significant difference $(p=0.915)$ in the mean pooled prevalence of SCIs between patients with $\mathrm{HbSC}$ and $\mathrm{HbS} \beta^{+} \mathrm{SCD}$ and controls (Fig. 3).

\section{$\mathrm{SCl}$ incidence}

Incidence data in different age groups was scarce, due to the inherent difficulties of longitudinal studies. Only four studies provided estimates ranging from 3.1 to $13.6 \%$ per

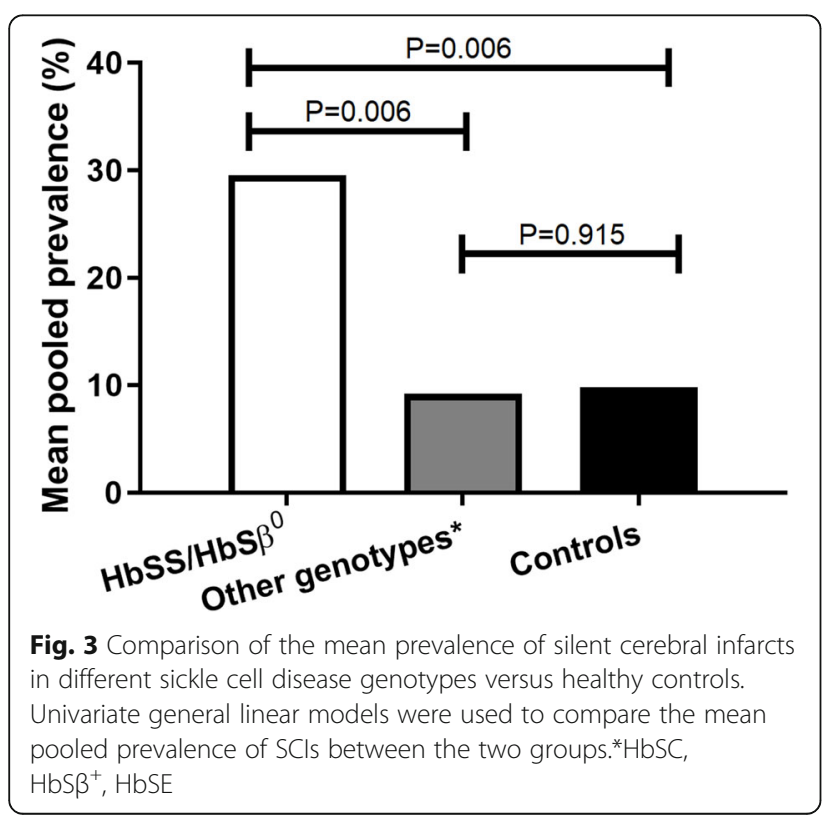

year $[10,23,34,43]$. In addition, the majority of studies did not address the first presentation of SCIs in young children, as most studies included patients older than 6 years of age because younger children often need sedation during MRI studies. However, four studies did report results from very young children, showing that SCIs begin to develop in children as young as 1 year of age $[6,54,55,59]$.

\section{Risk factors}

Thirty-three studies were identified that examined the risk factors for SCIs in patients with SCD (Table 3). Remarkably, for most risk factors, equal numbers of studies were found showing significant as well as insignificant associations. Furthermore, conflicting results were founded for two risk factors, i.e. mean systolic blood pressure and MCV with studies finding positive associations and studies finding negative associations.

Seven studies reported nine 'independent' risk factors in multivariable analyses (Table 4). Of these risk factors, only systolic blood pressure, haemoglobin level and foetal haemoglobin percentage were shown to be significant in more than one model. It was not possible to estimate a pooled odds ratio (OR) or a mean difference due to the small number of studies available.

\section{Clinical impact}

By definition, SCIs do not lead to overt neurological symptoms. However, they are associated with more subtle neurological deficits and an increased risk of subsequent overt stroke [10-12].

\section{Risk of cognitive decline}

Cognitive deficits have been demonstrated in patients with SCD using validated tests for general intelligence, visual processing and academic achievement. Several studies have reported poorer global intellectual function in patients with SCIs [12, 22, 26, 32, 38, 64, 69, 76, 77]. Of the nine included studies in which an association between lower cognitive test scores and SCIs was evaluated, such an association was found in eight. The CSSC D study showed that children with SCD and SCIs scored lower on full-scale intelligence quotient (IQ) $(p<0.003)$, verbal IQ $(p<0.01)$, reading $(p<0.04)$ and math achievement tests $(p<0.04)$, than children with normal MRI findings $[12,76]$.

Although overt stroke is an obvious cause of neurologic abnormality and cognitive impairment [12], cognitive deficits also occur in patients without evidence of focal brain injury [53, 69, 78-80]. The CSSCD study found that children with a persistently normal cerebral MRI during the entire 10-year study period still presented with a decline of 1.5 IQ points per year [76]. In addition, Hogan et al. showed that lower intellectual 


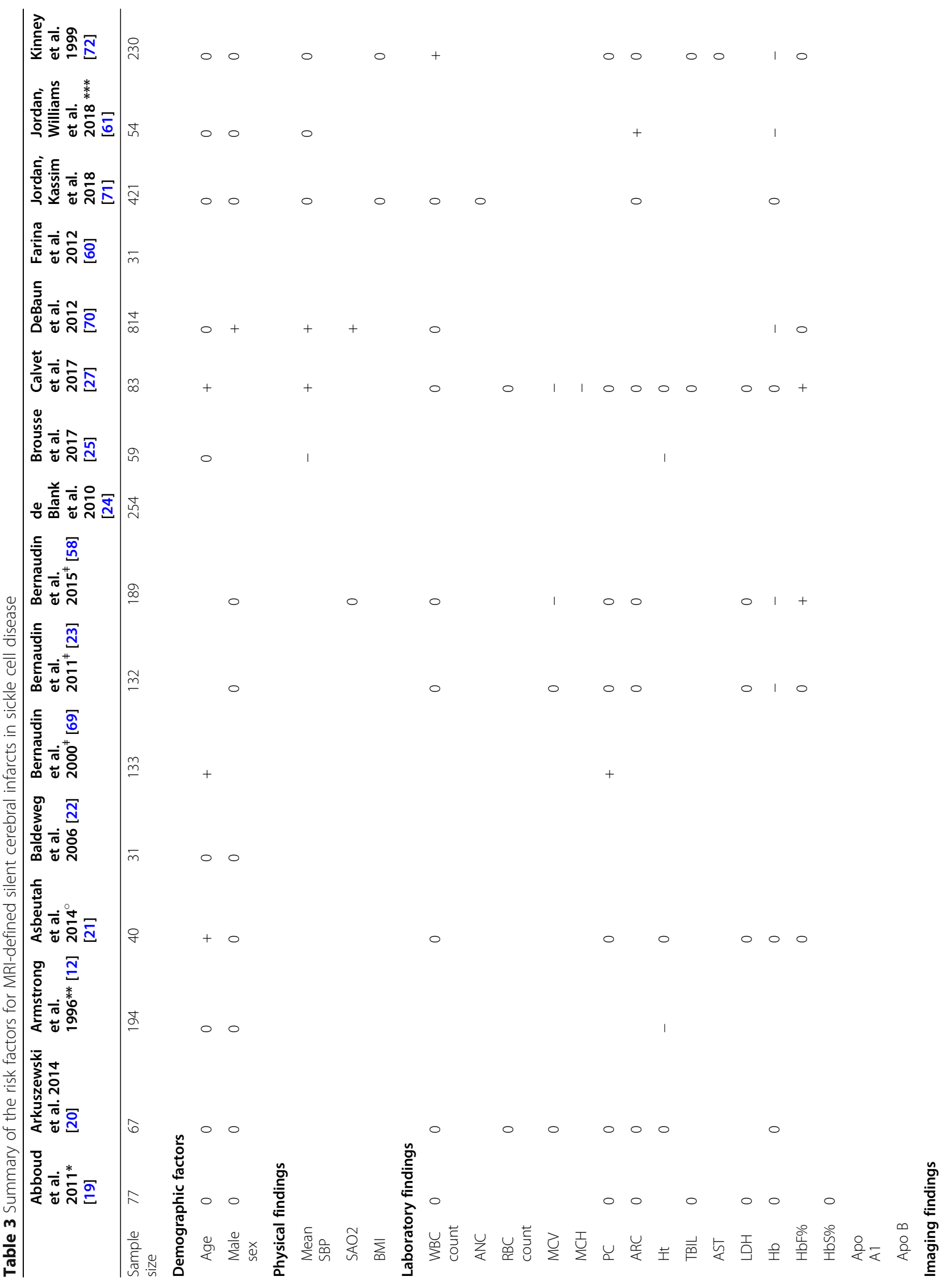




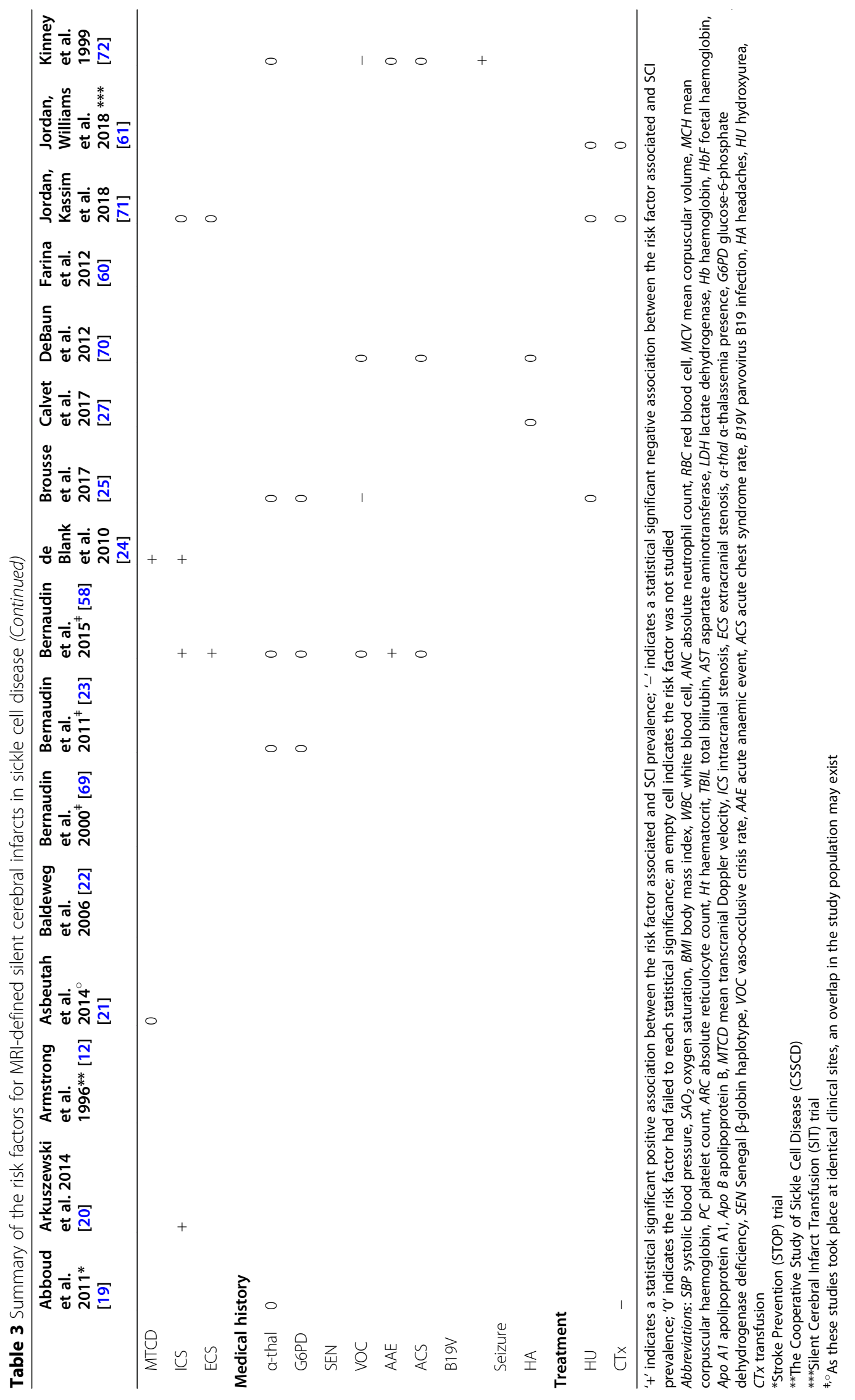




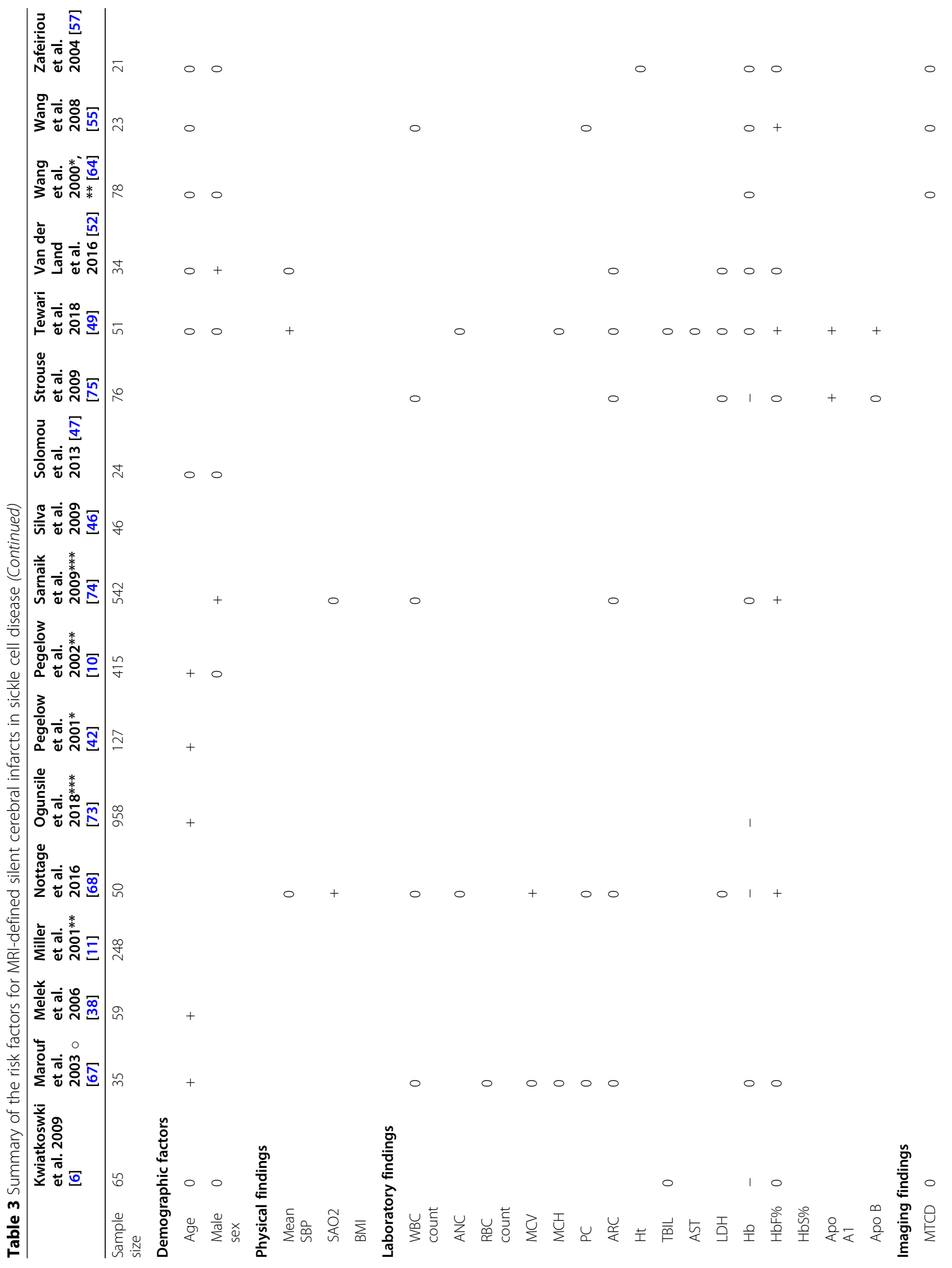




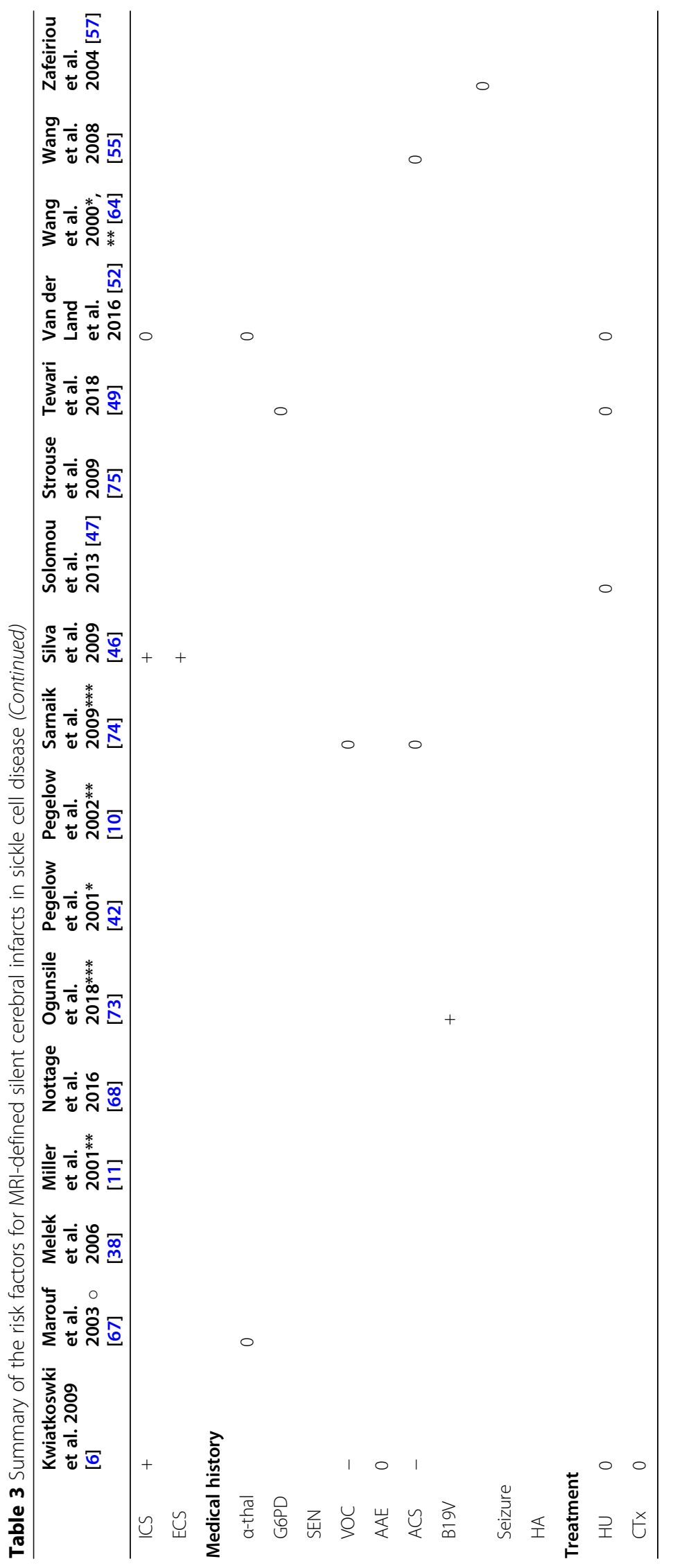


Table 4 Summary of the risk factors for MRI-defined silent cerebral infarcts in sickle cell disease analysed in multivariate models

\begin{tabular}{llll}
\hline Risk factors & OR/HR* $\mathbf{9 5 \%}$ Cl) & $\boldsymbol{p}$ value & Study \\
\hline Male sex & NA & 0.030 & DeBaun et al. 2012** [70] \\
Higher SBP & NA & 0.018 & DeBaun et al. 2012 $2^{* *}$ [70] \\
NA & $3.23(1.24-14.37)$ & 0.015 & Sarnaik et al. 2009 [74] \\
Higher WBC count & $1.75(1.14-2.78)$ & 0.016 & Kinney et al. 1999 [72] \\
Lower Hb level & $2.88(1.05-7.87)$ & 0.011 & Bernaudin et al. 2011 [23] \\
& NA & 0.039 & Bernaudin et al. 2015 [58] \\
Lower HbF\% & $0.84(0.72-0.97)$ & 0.001 & DeBaun et al. 2012** [70] \\
& NA & 0.02 & Calvet et al. 2017 [27] \\
Apolipoprotein A1 & 0.96 & 0.038 & Sarnaik et al. 2009 [74] \\
Extracranial stenosis & $3.11(1.10-8.85)$ & $<0.05$ & Strouse et al. 2009 [75] \\
SEN $\beta$-globin haplotype & $2.53(1.03-6.23)$ & 0.033 & Bernaudin et al. 2015 [58] \\
VOC rate & $0.53(0.30-0.95)$ & 0.044 & Kinney et al. 1999 [72] \\
Acute anaemic event & $3.39(1.01-11.34)$ & 0.034 & Kinney et al. 1999 [72] \\
Seizure & $14.4(1.5-141)$ & 0.048 & Bernaudin et al. 2015 [58] \\
\hline
\end{tabular}

Abbreviations: NA not available, SBP systolic blood pressure, WBC white blood cell, $H b$ haemoglobin, $H b F$ foetal haemoglobin, SEN Senegal, VOC vaso-occlusive crisis

*Results reported as odds ratio (OR) or hazard ratio (HR)

**This reduced multivariate logistic regression model was overall statistically significant $\left(x^{2}=24.41, \mathrm{df}=3, p=.001\right)$

functioning in children with SCD is partly explained by chronic hypoxia due to severe anaemia. This suggests that patients with normal MRI results may also have a constrained intellectual development [81]. Other studies conformingly show that low haemoglobin levels are a stronger predictor of neurocognitive function than SCIs [53, 69, 78].

\section{Risk of recurrent and progressive $\mathrm{SCls}$ or overt stroke}

SCIs are associated with an increased risk of subsequent stroke in patients with SCD. The CSSCD study was the first to report that the presence of SCIs is a risk factor for additional neurological injury, with a 14-fold increase in the risk of overt stroke, as $25 \%$ of children with SCIs presented with new or enlarged lesions at follow-up [10]. These findings have been confirmed in more recent publications, including in a study in which SCIs in very young children were associated with subsequent progressive ischaemia and a higher risk of overt stroke [59]. Further supportive of progressive ischaemia are the studies which report that many patients with SCIs present with more than one lesion $[20,21,27,28,32,33,36,50,77]$.

\section{Discussion}

\section{Challenges regarding reviewed studies}

Detection of SCIs is dependent upon the sensitivity and specificity of cerebral MRI scans and the definition of the radiological appearance. Importantly, advances in MRI technology lead to major heterogeneity when studying the prevalence, incidence and risk factors for the occurrence of SCIs in SCD. However, despite advancing neuroimaging technologies and therefore possible enhanced detection of SCIs, we did not find a rise in SCI prevalence in included studies over the years. MRI parameters varied widely in the studies included in this systematic review, with magnetic field strength ranging from 0.6 to $7.0 \mathrm{~T}$ and slice thickness ranging from 1.0 to $6.0 \mathrm{~mm}$. One study used 7-T MRI and identified many more intracerebral lesions when compared to 3-T MRI scanning, both in patients and in controls, with SCI prevalence rates as high as $90 \%$ and $70 \%$, respectively [51]. Although this study was excluded from our analysis as an outlier due to the extreme $Z$-score in order to not distort the overall analysis, it does suggest that SCI prevalence rates may actually be much higher when patients are screened with high-field magnet strength MRI. However, in accordance with the variability in SCI definitions utilized in the literature, the same study also observed that almost all lesions were smaller than $5 \mathrm{~mm}$, with a majority even smaller than $3 \mathrm{~mm}$ [51]. When so small, lesions may be easily confused with dilated perivascular spaces, also known as Virchow-Robin spaces, which may lead to an overall overestimation in prevalence [82].

Most studies did not define whether the performed MRI scans were reviewed with knowledge of patients' medical histories and whether or not neurological examination was performed by a neurologist. Because infarcts are classified as silent, if they-by definition-lack 
stroke-like symptoms, a haematologist may miss subtle neurological anomalies that may classify as a deficit in neurological examination by a neurologist.

We excluded studies with patients who had a history of overt stroke to ensure that no radiological apparent stroke was mistaken as SCIs. However, as SCIs have been identified as a risk factor for progression of SCIs and development of overt stroke, a large portion of stroke patients will also have additional SCIs. This was not taken into consideration in our analyses, and therefore, this exclusion criterion may have led to an underestimation in the prevalence of SCIs.

Most studies consisted of a heterogeneous group of patients. A few studies included individual patients on varying treatment regimens such as hydroxyurea medication or chronic red cell blood transfusions. Although currently there is no high-level evidence supporting these therapies as preventive for the development of SCIs [7], estimations of SCI prevalence may have been influenced by specific treatment regimens.

Finally, the absence of robust findings regarding the risk factors for the occurrence of SCIs is most probably due to the small patient sample sizes as well as weak associations. Tabulating the results in our systematic review was challenging due to the differences in definitions, heterogeneity of patients and treatment regimens and varying measurement methods and statistical analyses of many of the risk factors. Few studies explicitly stated the variables that were adjusted for in the multivariable analyses. In addition, the extent to which vascular risk factors are actually dependent upon one another is debatable, and interactions between the risk factors may have been overlooked by used analytic methods.

\section{Future directions for research}

The study limitations mentioned above provide several considerations for future studies. In particular, the use of a consistent definition of SCIs is crucial. We suggest a minimal MRI field strength of $1.5 \mathrm{~T}$ with 3 -mm-thick slices or thinner and at minimum the inclusion of FLAI $\mathrm{R}$ sequences. More specifically, lesions less than $3 \mathrm{~mm}$ in size should be excluded to minimize misdiagnosis of SCIs. In addition, reviewing of MRI scans as well as patient examination should be performed by experienced specialists.

Importantly, most studies were performed in children with SCD and limited attention has been given to adult patients. Longitudinal studies in patients over 16 years of age are necessary to understand the natural course of SCIs and their clinical relevance in adults with SCD. When designing future studies, it is essential to include matched control groups of healthy individuals, as siblings of patients with SCD often have sickle cell trait which is reported to possibly also impact cognition [48,
78, 83, 84]. Moreover, it is important to realize that healthy individuals also accumulate white matter hyperintensities with increasing age, which do not necessarily reflect SCIs. Epidemiological estimates have shown that the prevalence of SCIs detected by MRI screening is between 10 and $20 \%$ in the general population, with a strong association between SCI prevalence and age of the population assessed [82]. Unfortunately, the prevalence and incidence data on SCIs in populations younger than 45 years are lacking. Appreciation for possible SCIs in healthy children and young adults is essential to better understand SCI implications in SCD.

Lastly, further research is needed to determine the risk factors for and mechanisms of cognitive impairment in SCD in the absence of overt infarcts and SCIs. To this end, it is essential to differentiate between diseaserelated effects on brain function, indirect effects of chronic illnesses and psychosocial and socioeconomic factors [80]. This requires both longitudinal quantitative MRI and neuropsychological studies in combination with demographic and clinical variables.

\section{Conclusions}

SCIs are common in patients with $\mathrm{HbSS}$ and $\mathrm{HbS} \beta^{0}$ SCD with a weighted prevalence of $29.5 \%$. SCIs occur more often in patients with $\mathrm{HbSS}$ and $\mathrm{HbS} \beta^{0} \mathrm{SCD}$ when compared to other SCD genotypes and healthy controls, as respectively SCIs were found in $9.2 \%$ of $\mathrm{HbSC}$ and $\mathrm{HbS} \beta^{+}$patients and in $9.8 \%$ of controls.

Although the prevalence estimates varied widely across studies, data from this systematic review show a significant association between increasing patient age and SCI prevalence, which is consistent with an effect of age on cerebrovascular disease in SCD. Risk factor analyses showed no clear association between prevalence of SCIs and studied risk factors. Additional neuroimaging of patient populations with TCD, MRA or ASL may elucidate the pathogenesis and risk factors for the development of SCIs in SCD long term. Larger, prospective and controlled clinical, neuropsychological and neuroimaging studies are needed to understand how SCD and SCIs negatively affect cognition. Such studies may also provide a starting point for the identification of potential targets for preventive therapies by a better understanding of underlying pathophysiological mechanisms.

\section{Supplementary Information}

The online version contains supplementary material available at https://doi. org/10.1186/s12916-020-01864-8.

Additional file 1. Systematic search.

Additional file 2: Table 1. Prevalence of silent cerebral infarcts in control subjects. 


\section{Abbreviations}

ASL: Arterial spin labelling; CSSCD: Cooperative Study of Sickle Cell Disease: $\mathrm{CT}$ : Computed tomography; FLAIR: Fluid-attenuated inversion recovery; MRA: Magnetic resonance angiography; MRl: Magnetic resonance imaging; SCD: Sickle cell disease; SCIs: Silent cerebral infarctions; SIT trial: Silent Cerebral Infarct Transfusion trial; STOP trial: Stroke Prevention trial; TCD: Transcranial Doppler

\section{Acknowledgements}

NA

\section{Authors' contributions}

M.E.H., M.H.C. and T.J.H.W. designed the study. W.M.B. and M.E.H. designed and conducted the search strategy. M.E.H., R.L.G. and M.W.V screened the studies for eligibility, completed the data extraction and assessed the risk of bias. F.A. analysed the data. M.E.H. and R.L.G. wrote the manuscript in consultation with A.P.J.P. and C.M.Z. All authors discussed the results and contributed to the final manuscript. M.H.C. supervised the project. The authors read and approved the final manuscript.

\section{Funding}

NA

\section{Availability of data and materials}

The datasets used and/or analysed during the current study are available from the corresponding author on reasonable request.

\section{Ethics approval and consent to participate}

Not applicable (NA)

\section{Consent for publication}

NA

\section{Competing interests}

M.H. Cnossen has received investigator-initiated research and travel grants over the years from the Netherlands Organization for Scientific Research (NWO), the Netherlands Organization for Health Research and Development (ZonMw), the Dutch 'Innovatiefonds Zorgverzekeraars', Pfizer, Baxter/Baxalta/ Shire, Bayer Schering Pharma, CSL Behring, Sobi, Novo Nordisk, Novartis and Nordic Pharma and has served as a steering board member for Roche and Bayer. All grants, awards and fees go to the institution. P.J. de Pagter has received a grant from Rotary Foundation for the institution. All other authors declare no conflicts of interest relevant to the contents of this manuscript.

\section{Author details}

${ }^{1}$ Department of Pediatric Haematology and Oncology, Erasmus MC - Sophia Children's Hospital, NC-825, Wytemaweg 80, 3015 CN Rotterdam, The Netherlands. ${ }^{2}$ Department of Pediatric Radiology, Erasmus MC - Sophia Children's Hospital, Rotterdam, The Netherlands. ${ }^{3}$ Department of Haematology, Erasmus MC, Rotterdam, The Netherlands. ${ }^{4}$ Medical Library, Erasmus MC, Rotterdam, The Netherlands. ${ }^{5}$ Princess Máxima Center for Pediatric Oncology, Utrecht, The Netherlands. ${ }^{6}$ Department of Child and Adolescent Psychiatry, Erasmus MC - Sophia Children's Hospital, Rotterdam, The Netherlands. ${ }^{7}$ Department of Radiology and Nuclear Medicine, Erasmus MC, Rotterdam, The Netherlands. ${ }^{8}$ Department of Epidemiology, Erasmus MC, Rotterdam, The Netherlands.

Received: 30 July 2020 Accepted: 22 November 2020

Published online: 22 December 2020

\section{References}

1. Piel FB, Patil AP, Howes RE, Nyangiri OA, Gething PW, Dewi M, et al. Global epidemiology of sickle haemoglobin in neonates: a contemporary geostatistical model-based map and population estimates. Lancet. 2013; 381(9861):142-51.

2. Marotta CA, Forget BG, Cohne-Solal M, Wilson JT, Weissman SM. Human beta-globin messenger RNA. I. Nucleotide sequences derived from complementary RNA. J Biol Chem. 1977:252(14):5019-31.

3. Ataga Kl, Brittain JE, Desai P, May R, Jones S, Delaney J, et al. Association of coagulation activation with clinical complications in sickle cell disease. PLoS One. 2012;7(1):e29786
4. Manwani D, Frenette PS. Vaso-occlusion in sickle cell disease: pathophysiology and novel targeted therapies. Blood. 2013;122(24):3892-8.

5. DeBaun MR, Kirkham FJ. Central nervous system complications and management in sickle cell disease. Blood. 2016;127(7):829-38.

6. Kwiatkowski JL, Zimmerman RA, Pollock AN, Seto W, Smith-Whitley K, Shults $J$, et al. Silent infarcts in young children with sickle cell disease. $\mathrm{Br}$ J Haematol. 2009;146(3):300-5.

7. DeBaun MR, Armstrong FD, McKinstry RC, Ware RE, Vichinsky E, Kirkham FJ. Silent cerebral infarcts: a review on a prevalent and progressive cause of neurologic injury in sickle cell anemia. Blood. 2012;119(20): 4587-96.

8. Moser FG, Miller ST, Bello JA, Pegelow CH, Zimmerman RA, Wang WC, et al. The spectrum of brain MR abnormalities in sickle-cell disease: a report from the Cooperative Study of Sickle Cell Disease. AJNR Am J Neuroradiol. 1996; 17(5):965-72

9. Casella JF, King AA, Barton B, White DA, Noetzel MJ, Ichord RN, et al. Design of the silent cerebral infarct transfusion (SIT) trial. Pediatr Hematol Oncol. 2010;27(2):69-89.

10. Pegelow CH, Macklin EA, Moser FG, Wang WC, Bello JA, Miller ST, et al. Longitudinal changes in brain magnetic resonance imaging findings in children with sickle cell disease. Blood. 2002:99(8):3014-8.

11. Miller ST, Macklin EA, Pegelow CH, Kinney TR, Sleeper LA, Bello JA, et al. Silent infarction as a risk factor for overt stroke in children with sickle cell anemia: a report from the Cooperative Study of Sickle Cell Disease. J Pediatr. 2001;139(3):385-90.

12. Armstrong FD, Thompson RJ Jr, Wang W, Zimmerman R, Pegelow CH, Miller $\mathrm{S}$, et al. Cognitive functioning and brain magnetic resonance imaging in children with sickle cell disease. Neuropsychology Committee of the Cooperative Study of Sickle Cell Disease. Pediatrics. 1996;97(6 Pt 1):864-70.

13. Vermeer SE, Longstreth WT Jr, Koudstaal PJ. Silent brain infarcts: a systematic review. Lancet Neurol. 2007;6(7):611-9.

14. Yawn BP, Buchanan GR, Afenyi-Annan AN, Ballas SK, Hassell KL, James AH, et al. Management of sickle cell disease: summary of the 2014 evidencebased report by expert panel members. JAMA. 2014;312(10):1033-48.

15. DeBaun MR. The challenge of creating an evidence-based guideline for sickle cell disease. Jama. 2014;312(10):1004-5.

16. DeBaun MR, Gordon M, McKinstry RC, Noetzel MJ, White DA, Sarnaik SA, et al. Controlled trial of transfusions for silent cerebral infarcts in sickle cell anemia. N Engl J Med. 2014;371(8):699-710.

17. Moher D, Shamseer L, Clarke M, Ghersi D, Liberati A, Petticrew M, et al. Preferred Reporting Items for Systematic Review and Meta-Analysis Protocols (PRISMA-P) 2015 Statement. Syst Rev. 2015;4:1.

18. Forbes K. MRI brain white matter change: spectrum of change - how we can grade? J R Coll Physicians Edinb. 2017;47(3):271-5.

19. Abboud MR, Yim E, Musallam KM, Adams RJ, Investigators SIS. Discontinuing prophylactic transfusions increases the risk of silent brain infarction in children with sickle cell disease: data from STOP II. Blood. 2011;118(4):894-8.

20. Arkuszewski M, Krejza J, Chen R, Ichord R, Kwiatkowski JL, Bilello M, et al. Sickle cell anemia: intracranial stenosis and silent cerebral infarcts in children with low risk of stroke. Adv Med Sci. 2014;59(1):108-13.

21. Asbeutah A, Gupta R, Al-Saeid O, Ashebu S, Al-Sharida S, Mullah-Ali A, et al. Transcranial Doppler and brain MRI in children with sickle cell disease and high hemoglobin F levels. Pediatr Blood Cancer. 2014;61(1):25-8.

22. Baldeweg T, Hogan AM, Saunders DE, Telfer P, Gadian DG, Vargha-Khadem $F$, et al. Detecting white matter injury in sickle cell disease using voxelbased morphometry. Ann Neurol. 2006;59(4):662-72.

23. Bernaudin F, Verlhac S, Arnaud C, Kamdem A, Chevret S, Hau I, et al. Impact of early transcranial Doppler screening and intensive therapy on cerebral vasculopathy outcome in a newborn sickle cell anemia cohort. Blood. 2011; 117(4):1130-40 quiz 436.

24. de Blank PMK, Hayward DM, Zimmerman MDR, Pollock A, Kwiatkowski JL. Transcranial Doppler ultrasound velocity, cerebral vasculopathy, and silent infarcts in sickle cell disease. Blood. 2010;116(21):269.

25. Brousse V, Grévent D, Arnaud C, Kamden A, Kossorotoff M, De Montalembert M, et al. Silent cerebral infarct pathophysiology study in sickle cell anemia children by a multimodal approach. Blood. 2017; 130(Suppl 1):3532.

26. Brown RT, Davis PC, Lambert R, Hsu L, Hopkins K, Eckman J. Neurocognitive functioning and magnetic resonance imaging in children with sickle cell disease. J Pediatr Psychol. 2000;25(7):503-13. 
27. Calvet D, Tuilier T, Mele N, Turc G, Habibi A, Abdallah NA, et al. Low fetal hemoglobin percentage is associated with silent brain lesions in adults with homozygous sickle cell disease. Blood Adv. 2017;1(26):2503-9.

28. Coloigner J, Phlypo R, Coates TD, Lepore N, Wood JC. Graph lasso-based test for evaluating functional brain connectivity in sickle cell disease. Brain Connect. 2017:7(7):443-53.

29. Dowling MM, Quinn CT, Plumb P, Rogers ZR, Rollins NK, Koral K, et al. Acute silent cerebral ischemia and infarction during acute anemia in children with and without sickle cell disease. Blood. 2012;120(19):3891-7.

30. Ford AL, Fields ME, Guilliams KP, Ragan DK, Yasheng C, Guillaume G, et al. Increased volume and distinct pattern of silent cerebral infarcts in healthy, young adults with sickle cell trait. Blood. 2017;130(Suppl 1):757.

31. Ford AL, Ragan DK, Fellah S, Binkley MM, Fields ME, Guilliams KP, et al. Silent infarcts in sickle cell disease occur in the border zone region and are associated with low cerebral blood flow. Blood. 2018;132(16):1714-23.

32. Gold Jl, Johnson CB, Treadwell MJ, Hans N, Vichinsky E. Detection and assessment of stroke in patients with sickle cell disease: neuropsychological functioning and magnetic resonance imaging. Pediatr Hematol Oncol. 2008; 25(5):409-21.

33. Guilliams KP, Fields ME, Hulbert ML. Higher-than-expected prevalence of silent cerebral infarcts in children with hemoglobin SC disease. Blood. 2015; 125(2):416-7.

34. Gyang E, Yeom K, Hoppe C, Partap S, Jeng M. Effect of chronic red cell transfusion therapy on vasculopathies and silent infarcts in patients with sickle cell disease. Am J Hematol. 2011;86(1):104-6.

35. Issar $P$, Nehra M, Singh G, Issar SK. Conventional and advanced brain MR imaging in patients with sickle cell anemia. Indian J Radiol Imaging. 2018; 28(3):305-11.

36. Kassim AA, Pruthi S, Day M, Rodeghier M, Gindville MC, Brodsky MA, et al. Silent cerebral infarcts and cerebral aneurysms are prevalent in adults with sickle cell anemia. Blood. 2016;127(16):2038-40.

37. Kawadler JM, Hales PW, Barker S, Cox TCS, Kirkham FJ, Clark CA. Cerebral perfusion characteristics show differences in younger versus older children with sickle cell anaemia: results from a multiple-inflow-time arterial spin labelling study. NMR Biomed. 2018;31(6):e3915.

38. Melek I, Akgul F, Duman T, Yalcin F, Gali E. Neurological soft signs as the stroke risk in sickle cell disease. Tohoku J Exp Med. 2006;209(2):135-40.

39. Mercuri E, Faundez JC, Roberts I, Flora S, Bouza H, Cowan F, et al. Neurological 'soft' signs may identify children with sickle cell disease who are at risk for stroke. Eur J Pediatr. 1995;154(2):150-6.

40. Kader KO, Xavier G, Francesca BP, Catherine AF, Nevada W, Rebecca I, et al Sickle cell disease: continuous arterial spin-labeling perfusion MR imaging in children. Radiology. 2003;227(2):567-74.

41. Onofri A, Montanaro M, Rampazzo P, Colombatti R, Farina FM, Manara R, et al. Intellectual impairment and TCD evaluation in children with sickle cell disease and silent stroke. Perspectives Med. 2012;1(1):272-4

42. Pegelow CH, Wang W, Granger S, Hsu LL, Vichinsky E, Moser FG, et al. Silent infarcts in children with sickle cell anemia and abnormal cerebral artery velocity. Arch Neurol. 2001;58(12):2017-21.

43. Quinn CT, McKinstry RC, Dowling MM, Ball WS, Kraut MA, Casella JF, et al. Acute silent cerebral ischemic events in children with sickle cell anemia. JAMA Neurol. 2013;70(1):58-65.

44. Schatz J, Buzan R. Decreased corpus callosum size in sickle cell disease: relationship with cerebral infarcts and cognitive functioning. J Int Neuropsychol Soc. 2006;12(1):24-33.

45. Seibert JJ, Miller SF, Kirby RS, Becton DL, James CA, Glasier CM, et al. Cerebrovascular disease in symptomatic and asymptomatic patients with sickle cell anemia: screening with duplex transcranial Doppler US-correlation with MR imaging and MR angiography. Radiology. 1993; 189(2):457-66

46. Silva GS, Vicari P, Figueiredo MS, Carrete $H$ Jr, Idagawa MH, Massaro AR. Brain magnetic resonance imaging abnormalities in adult patients with sickle cell disease: correlation with transcranial Doppler findings. Stroke. 2009;40(7):2408-12.

47. Solomou E, Kraniotis P, Kourakli A, Petsas T. Extent of silent cerebral infarcts in adult sickle-cell disease patients on magnetic resonance imaging: is there a correlation with the clinical severity of disease? Hematol Rep. 2013;5(1):8-12.

48. Steen RG, Emudianughe T, Hankins GM, Wynn LW, Wang WC, Xiong X, et al. Brain imaging findings in pediatric patients with sickle cell disease. Radiology. 2003;228(1):216-25.
49. Tewari S, Renney G, Brewin J, Gardner K, Kirkham F, Inusa B, et al. Proteomic analysis of plasma from children with sickle cell anemia and silent cerebral infarction. Haematologica. 2018;103(7):1136-42.

50. Vaclavu L, Meynart BN, Mutsaerts H, Petersen ET, Majoie C, VanBavel ET, et al. Hemodynamic provocation with acetazolamide shows impaired cerebrovascular reserve in adults with sickle cell disease. Haematologica. 2019;104(4):690-9.

51. van der Land V, Zwanenburg JJ, Fijnvandraat K, Biemond BJ, Hendrikse J, Mutsaerts $\mathrm{HJ}$, et al. Cerebral lesions on 7 tesla MRI in patients with sickle cell anemia. Cerebrovasc Dis. 2015;39(3-4):181-9.

52. van der Land V, Mutsaerts HJ, Engelen M, Heijboer $\mathrm{H}$, Roest M, Hollestelle $\mathrm{MJ}$, et al. Risk factor analysis of cerebral white matter hyperintensities in children with sickle cell disease. Br J Haematol. 2016;172(2):274-84.

53. Vichinsky EP, Neumayr LD, Gold JI, Weiner MW, Rule RR, Truran D, et al. Neuropsychological dysfunction and neuroimaging abnormalities in neurologically intact adults with sickle cell anemia. Jama. 2010;303(18): 1823-31.

54. Wang WC, Langston JW, Steen RG, Wynn LW, Mulhern RK, Wilimas JA, et al. Abnormalities of the central nervous system in very young children with sickle cell anemia. J Pediatr. 1998;132(6):994-8.

55. Wang WC, Pavlakis SG, Helton KJ, McKinstry RC, Casella JF, Adams RJ, et al. MRI abnormalities of the brain in one-year-old children with sickle cell anemia. Pediatr Blood Cancer. 2008;51(5):643-6.

56. Watkins KE, Hewes DK, Connelly A, Kendall BE, Kingsley DP, Evans JE, et al. Cognitive deficits associated with frontal-lobe infarction in children with sickle cell disease. Dev Med Child Neurol. 1998;40(8):536-43.

57. Zafeiriou DI, Prengler M, Gombakis N, Kouskouras K, Economou M, Kardoulas A, et al. Central nervous system abnormalities in asymptomatic young patients with S $\beta$-thalassemia. Ann Neurol. 2004; 55(6):835-9.

58. Bernaudin F, Verlhac S, Arnaud C, Kamdem A, Vasile M, Kasbi F, et al. Chronic and acute anemia and extracranial internal carotid stenosis are risk factors for silent cerebral infarcts in sickle cell anemia. Blood. 2015; 125(10):1653-61.

59. Cancio MI, Helton KJ, Schreiber JE, Smeltzer MP, Kang G, Wang WC. Silent cerebral infarcts in very young children with sickle cell anaemia are associated with a higher risk of stroke. Br J Haematol. 2015;171(1):120-9.

60. Farina FM, Rampazzo P, Sainati L, Manara R, Onofri A, Colombatti R, et al. Transcranial Doppler sonography in children with sickle cell disease and silent ischemic lesions. Perspectives Med. 2012;1(1):269-71.

61. Jordan LC, Roberts Williams DO, Rodeghier MJ, Covert Greene BV, Ponisio MR, Casella JF, et al. Children with sickle cell anemia with normal transcranial Doppler ultrasounds and without silent infarcts have a low incidence of new strokes. Am J Hematol. 2018:93(6):760-8.

62. Kral MC, Brown RT, Curé JK, Besenski N, Jackson SM, Abboud MR. Radiographic predictors of neurocognitive functioning in pediatric sickle cell disease. J Child Neurol. 2006:21(1):37-44.

63. Thangarajh M, Yang G, Fuchs D, Ponisio MR, McKinstry RC, Jaju A, et al. Magnetic resonance angiography-defined intracranial vasculopathy is associated with silent cerebral infarcts and glucose-6-phosphate dehydrogenase mutation in children with sickle cell anaemia. $\mathrm{Br}$ Haematol. 2012;159(3):352-9.

64. Wang WC, Gallagher DM, Pegelow CH, Wright EC, Vichinsky EP, Abboud MR, et al. Multicenter comparison of magnetic resonance imaging and transcranial Doppler ultrasonography in the evaluation of the central nervous system in children with sickle cell disease. J Pediatr Hematol Oncol. 2000:22(4):335-9.

65. Gevers S, Nederveen AJ, Fijnvandraat K, van den Berg SM, van Ooij P, Heijtel $D F$, et al. Arterial spin labeling measurement of cerebral perfusion in children with sickle cell disease. J Magn Reson Imaging. 2012;35(4):779-87.

66. Helton KJ, Paydar A, Glass J, Weirich EM, Hankins J, Li C-S, et al. Arterial spinlabeled perfusion combined with segmentation techniques to evaluate cerebral blood flow in white and gray matter of children with sickle cell anemia. Pediatr Blood Cancer. 2009;52(1):85-91.

67. Marouf R, Gupta R, Haider MZ, Adekile AD. Silent brain infarcts in adult Kuwaiti sickle cell disease patients. Am J Hematol. 2003;73(4):240-3.

68. Nottage KA, Ware RE, Aygun B, Smeltzer M, Kang G, Moen J, et al. Hydroxycarbamide treatment and brain MRI/MRA findings in children with sickle cell anaemia. Br J Haematol. 2016;175(2):331-8.

69. Bernaudin F, Verlhac S, Freard F, Roudot-Thoraval F, Benkerrou M, Thuret I, et al. Multicenter prospective study of children with sickle cell 
disease: radiographic and psychometric correlation. J Child Neurol. 2000; 15(5):333-43.

70. DeBaun MR, Sarnaik SA, Rodeghier MJ, Minniti CP, Howard TH, lyer RV, et al. Associated risk factors for silent cerebral infarcts in sickle cell anemia: low baseline hemoglobin, sex, and relative high systolic blood pressure. Blood. 2012;119(16):3684-90.

71. Jordan LC, Kassim AA, Donahue MJ, Juttukonda MR, Pruthi S, Davis LT, et al. Silent infarct is a risk factor for infarct recurrence in adults with sickle cell anemia. Neurology. 2018;91(8):e781-e4.

72. Kinney TR, Sleeper LA, Wang WC, Zimmerman RA, Pegelow CH, OheneFrempong $\mathrm{K}$, et al. Silent cerebral infarcts in sickle cell anemia: a risk factor analysis. The Cooperative Study of Sickle Cell Disease. Pediatrics. 1999;103(3):640-5.

73. Ogunsile FJ, Currie KL, Rodeghier M, Kassim A, DeBaun MR, Sharma D. History of parvovirus B19 infection is associated with silent cerebral infarcts. Pediatr Blood Cancer. 2018;65(1):e26767.

74. Sarnaik SA, Casella JF, Barton BA, Afif M, Airewele G, Berman BW, et al. Elevated systolic blood pressure and low fetal hemoglobin are risk factors for silent cerebral infarcts in children with sickle cell anemia. Blood. 2009; 114(22):262

75. Strouse JJ, Kraut M, Williams LM, DeBaun MR, Casella JF. Low levels of apolipoprotein A1 are associated with silent cerebral infarcts in children with sickle cell disease. Blood. 2009;114(22):259.

76. Wang W, Enos L, Gallagher D, Thompson R, Guarini L, Vichinsky E, et al. Neuropsychologic performance in school-aged children with sickle cell disease: a report from the Cooperative Study of Sickle Cell Disease. J Pediatr. 2001;139(3):391-7.

77. van der Land V, Hijmans $C T$, de Ruiter M, Mutsaerts HJMM, Cnossen MH, Engelen $\mathrm{M}$, et al. Volume of white matter hyperintensities is an independent predictor of intelligence quotient and processing speed in children with sickle cell disease. Br J Haematol. 2015;168(4):553-6.

78. Steen RG, Fineberg-Buchner C, Hankins G, Weiss L, Prifitera A, Mulhern RK. Cognitive deficits in children with sickle cell disease. J Child Neurol. 2005; 20(2):102-7.

79. Kawadler JM, Clayden JD, Clark CA, Kirkham FJ. Intelligence quotient in paediatric sickle cell disease: a systematic review and meta-analysis. Dev Med Child Neurol. 2016:58(7):672-9.

80. Schatz J, Finke RL, Kellett JM, Kramer JH. Cognitive functioning in children with sickle cell disease: a meta-analysis. J Pediatr Psychol. 2002;27(8):739-48.

81. Hogan AM, Pit-ten Cate IM, Vargha-Khadem F, Prengler M, Kirkham FJ. Physiological correlates of intellectual function in children with sickle cell disease: hypoxaemia, hyperaemia and brain infarction. Dev Sci. 2006;9(4):379-87.

82. Fanning JP, Wong AA, Fraser JF. The epidemiology of silent brain infarction: a systematic review of population-based cohorts. BMC Med. 2014;12:119.

83. Caughey MC, Loehr LR, Key NS, Derebail VK, Gottesman RF, Kshirsagar AV, et al. Sickle cell trait and incident ischemic stroke in the Atherosclerosis Risk in Communities study. Stroke. 2014;45(10):2863-7.

84. Cahill CR, Leach JM, McClure LA, Irvin MR, Zakai NA, Naik R, et al. Sickle cell trait and risk of cognitive impairment in African-Americans: the REGARDS cohort. EClinicalMedicine. 2019;11:27-33.

\section{Publisher's Note}

Springer Nature remains neutral with regard to jurisdictional claims in published maps and institutional affiliations.

Ready to submit your research? Choose BMC and benefit from:

- fast, convenient online submission

- thorough peer review by experienced researchers in your field

- rapid publication on acceptance

- support for research data, including large and complex data types

- gold Open Access which fosters wider collaboration and increased citations

- maximum visibility for your research: over $100 \mathrm{M}$ website views per year

At BMC, research is always in progress.

Learn more biomedcentral.com/submissions 